\title{
Suppression of a learned response by punishment when an alternative learned response is provided ${ }^{\prime 2}$
}

JAMES L. BRUNING, B. L. KINTZ, AND MORRIS K. MORGRET

OHIO UNIVERSITY

\begin{abstract}
Three groups of 16 rats were trained in a $\mathrm{T}$-maze which contained a large reward in one arm and a small reward in the other. A forced choice technique insured that all Ss entered each arm of the maze an equal proportion of the time. After the Ss had learned to correctly choose the large reward side, the Ss in Group 1 were given a 1 sec. mild shock (.2 ma) upon entering the large reward goal box, Group 2 a 1 sec. strong shock (1.2 ma) and Group 3 a .2 ma shock which was gradually increased by .1 ma on each succeeding block of six trials. The results indicated that: (1) During acquisition the Ss showed great reluctance to enter the arm containing small reward. (2) Presentation of shock to the Ss in Groups 2 and 3 suppressed the approach tendency to the large reward side. However, the Ss also refused to go to the arm containing small reward.
\end{abstract}

\section{Introduction}

Association of reward and punishment with the same goal has been viewed by many psychologists as a primary factor in the development of neuroses by humans. Similarly, the disruptive effects of such an approachavoidance conflict on animal behavior has been demonstrated rather dramatically by Masserman (1943).

However, such complete disruption of normal behavior does not always result. In a much quoted bar-press experiment by Estes (1944), the effects of punishment were shown to be only temporary. In addition to the Estes experiment there are human (Sears, Maccoby, \& Levin, 1957) and animal (Muenzinger, Bernstone, \& Richards, 1938) studies which show that punishment actually increases the tendency to approach the goal. Thus, on the basis of the above findings, a reasonable conclusion might be that punishment is, at best, ineffective in changing behavior and, at worst, results in various forms of abnormal behavior.

One finding contrary to this conclusion is reported by Whiting \& Mowrer (1943). In their experiment a maze was used which had a long and short path to food. After the animals had learned to take the short path, three experimental conditions were imposed. The Ss in Group 1 were rewarded only if they took the long path, Group 2 had a barrier placed in the short path, while Group 3 was punished for taking the short path. The groups took 230, 82, and 6 trials, respectively, to change from choosing the shorter path. Thus, punishment was shown to be very effective in changing behavior when an alternative, although less attractive, response was available and reinforced. The present study is similar to that of Whiting and Mowrer except that the parameter varied was size of reward rather than length of the alley.

\section{Method}

Forty-eight Sprague-Dawley rats were trained in a T-maze which had an electrifiable grid floor. The stem and each of the arms was $3 \mathrm{ft}$ long. The stem was painted flat gray, the right arm was black, and the left one white.

The Ss were maintained on a $22 \mathrm{hr}$. food deprivation schedule. For half of the Ss, five Noyes pellets were placed in the black arm of the maze and one pellet in the white arm. For the other half, the placing of the large and small reward was reversed to control for color and position effects. Six trials were given per day, three of which were "free choice" and three "forced" to insure that the Ss went to each of the goal boxes an equal number of times. On forced trials a guillotine door separating the large reward arm of the maze from the stem was closed. In addition to the arm chosen by the $\mathrm{S}$ on the free choice trials, two measures of response latency were taken. The first was taken from the time the start door was opened until the $S$ had moved one foot down the stem of the maze. The second measure, the "decision time," was the time taken by the $S$ to move through the choice point and begin entry into either of the goal boxes. A criterion of $3 \mathrm{~min}$. was set as the maximum length of any particular trial.

After the Ss had learned to consistently choose the large reward arm, they were divided into three groups of 16 each. The Ss in Groups 1 and 2 experienced 1 sec. of shock just as they reached the large reward goal box. Since the shock was associated only with large reward, the Ss could go to the opposite arm which had no shock, but of course had to accept a lesser amount of food. For Group 1, the shock was set at only $.2 \mathrm{ma}$ of constant current. This is barely sufficient to elicit a flinch or twitch response in the animal. For Group 2, the intensity was set at $1.2 \mathrm{ma}$ which causes considerable agitation and apparent pain. Group 3 was originally designated as a control group that was to receive the test trials under exactly the same conditions experienced during acquisition, except that all trials were to be free choice.

\section{Results and Diseussion}

Considering first the measures recorded during acquisition, it was noted that by the 24th trial, all Ss had met the criterion of 10 out of 10 correct choices 
of the large reward side. Of particular interest was the difference in the behavior of the Ss on the free vs. forced choice trials. On the free choice trials the Ss went immediately to the large reward. However, when the arm of the maze leading to large reward was blocked off, the Ss showed considerable vacillation and reluctance to enter the small reward arm. A statistical analysis of the decision times on the free and forced choice trials indicated that the difference was significant well beyond the .001 level $(F=33.75$, $\mathrm{df}=1 / 47) .3$

The most reasonable interpretation of this sizable difference seems to be, as Denny (1955) has suggested, that after the $\mathrm{S}$ has previously experienced larger reward, a smaller amount serves as a mild noxious stimulus and elicits avoidance tendencies. Consequently, the $\mathrm{S}$ becomes reticent to enter the arm which contains smaller reward.

During the test phase of the experiment, when the shocks were administered to the various groups, it was noted that instead of running faster toward the large reward side, as would be expected on the basis of Muenzinger's (1938) research, the Ss in Group 1 showed a slight, but nonsignificant tendency to slow down $(\mathrm{F}=1.72$, $\mathrm{df}=4 / 60)$.

Of greater interest was the behavior of the Ss in Group 2. After only one or two shocks, all of these Ss stopped running, not only to the large reward side, but also refuised to go to the small reward side on any of the 24 test trials. Observation of their behavior suggested that the high intensity of the shock may have resulted in such a generalized fear that the tendency to approach in either arm was suppressed. To test this hypothesis, the Group $3 \mathrm{Ss}$, who originally were to have served as controls, were submitted to a condition in which they initially received a shock in the large reward goal box equal to that received by the Ss in Group 1, e.g., .2 ma. On each succeeding block of six trials, the intensity was increased by .1 ma until the Ss stopped approaching the large reward side. It was reasoned that by increasing the shock intensity across several trials, the avoidance tendency would gradually generalize from the goal box to the choice point and the Ss would begin choosing the smaller reward. However, the results indicated that when the shock be- came strong enough to suppress the approach tendency to the large reward side (in no instance was a shock as intense as $1.2 \mathrm{ma}$ needed), all but one of the Ss refused to run and would not enter the small reward side.

These findings were somewhat surprising, but seem quite reasonable if Denny's assumptions are considered that avoidance tendencies result when small reward follows the experiencing of larger amounts. Since there was shock in one arm of the maze and an unwanted small reward in the other, the Ss in Groups 2 and 3 were faced with an avoidance-avoidance conflict which they resolved by staying in the stem of the maze.

The results of this investigation indicate, first, that the statement which currently appears in several introductory and child psychology books that punishment is effective in breaking a habit only when another habit is provided and reinforced, must be further qualified to include reference to specific situations. Second, the assumptions made by Spence (1956) that delay of reward and size of reward affect incentive in the same fashion must also be qualified, since it appears that small reward can acquire negative reinforcing characteristics, while a long delay, at least in the form of a long alley, apparently does not elicit avoidance tendencies.

\section{References}

Denny, M. R. \& King, G. F. Differential response learning on the basis of differential size of reward. J. genet. Psychol., 1955 87, $317-320$.

Estes, W. K. An experimental study of punishment. Psychol. Monogr., 1944, 57, No. 263.

Masserman, J. H. Behavior and neurosis. Chicago: University of Chicago Press, 1943.

Meunzinger, K. F., Bernstone, A. H., \& Richards, L. Motivation in in learning: VIII. Equivalent amounts of electric shock for right and wrong responses in a visual discrimination habit. J. comp. physiol. Psychol., 1938, 26, 177-186.

Sears, R. R., Maccoby, E. E., \& Levine, H. Patterns of child rearing. White Plains, N. Y.: Row, Peterson, 1957.

Spence, K. W. Behavior theory and conditioning. New Haven: Yale University Press, 1956.

Whiting, J. W., \& Mowrer, O. H. Habit progression and regression-a laboratory investigation of some factors relevant to human socialization. J. comp. Psychol., 1943, 36, 229-253.

\section{Notes}

1. The contents of this paper were presented at the Midwestern Psychological Association convention in April, 196

2. This study was supported in part by the Ohio I ersity Research Committee.

3. The start speed measure proved to be of little value in this study. The effects of interest occurred in the region of the choice point which was more contiguous with both the reward and punishment. 